\title{
Repositórios educacionais: uma análise da usabilidade do eduCAPES
}

Isadora Lopes Barbosa Vasconcellos - IFFluminense - isadora.vasconcellos@ gmail.com Angelina Barros Mota Arêas - IFFluminense - angel_areas@ hotmail.com Jéssica Raquel Pereira Ribeiro - IFFluminense - jraquelribeiro@gmail.com Soraya Castro de Lima Oliveira - IFFluminense - sorayacastrodelima@ gmail.com Arilise Moraes de Almeida Lopes - IFFluminense - arilise@iff.edu.br

Resumo: Este artigo objetiva analisar a usabilidade de um repositório educacional instituído pelo Governo Federal, o eduCAPES, que reúne, compartilha e organiza objetos de aprendizagem. Para tanto, foi realizada uma análise documental com o propósito de identificar trabalhos que apresentassem informações sobre os conceitos e critérios de usabilidade em repositórios educacionais. A partir desta pesquisa, foram estabelecidos 28 critérios, divididos em cinco categorias, para analisar a usabilidade do eduCAPES. Como resultado, apesar de a maioria dos critérios apresentar aspectos positivos, constatou-se que mudanças podem ser implementadas. Espera-se, com esta análise, a partir da inclusão das melhorias propostas, potencializar a satisfação e a percepção da eficiência em relação ao uso do eduCAPES pela comunidade acadêmica.

Palavras-Chaves: repositório, eduCAPES, usabilidade.

\section{Educational repositories: an analysis of the usability of eduCAPES}

Abstract: This article aims to analyze the usability of an educational repository instituted by the Federal Government, the eduCAPES, which gathers, shares and organizes learning objects. Therefore, a documentary analysis was used with the purpose of identifying works that presented information about the concepts and criteria of usability in educational repositories. From this research, 28 criteria were established, divided into five categories, to analyze the usability of eduCAPES. As a result, although most of the criteria present positive aspects, it was observed that changes can be implemented. It is hoped, with this analysis, from the inclusion of the proposed improvements, to enhance the satisfaction and the perception of efficiency in relation to the use of eduCAPES by the academic community.

Keywords: repository, eduCAPES, usability.

\section{Introdução}

A popularização da Internet possibilitou a disponibilidade de uma infinidade de informações que podem ser, dentre outras coisas, utilizadas com fins educativos, desde que sejam selecionadas e/ou elaboradas de acordo com os objetivos educacionais a que se destinam. Dentre as tecnologias que surgiram devido à necessidade de compartilhar informações na Internet, estão as plataformas colaborativas. Estas, ao serem utilizadas na área de educação, viabilizam a comunicação e a colaboração, permitindo o compartilhamento de recursos didáticos (Almeida; Freitas, 2015).

Um exemplo dessas plataformas são os repositórios educacionais, que têm como propósito reunir, compartilhar e organizar recursos didáticos disponíveis na Internet (Pinheiro et al., 2016). Os materiais disponibilizados nos repositórios educacionais recebem a classificação de Objetos de Aprendizagem (OA), uma vez que são recursos utilizados com o propósito de ensino e aprendizagem. Porém, para que sejam úteis no processo educacional, é necessário o reconhecimento, por parte do professor, de suas 
potencialidades e limitações na aplicação dos conceitos explorados em função do objetivo proposto (Bonotto; Bisognin, 2015).

De acordo com Pinheiro et al. (2016), são considerados OA quaisquer recursos digitais usados com objetivo educacional. Assim, a função básica de um repositório educacional é permitir o acesso aos OA nele armazenados de forma organizada e sistemática (Sabbatini, 2012). Desta forma, os repositórios educacionais apresentam OA e seus metadados, que são informações que descrevem os OA com o objetivo de facilitar e tornar mais eficaz a recuperação destes (Sabbatini, 2012).

Os professores, ao utilizarem um repositório educacional, devem considerar alguns aspectos, tais como: a facilidade de busca, as informações sobre os OA, os tipos de OA que são disponibilizados, qual o conteúdo e público-alvo, se os OA são atrativos e fáceis de serem utilizados, entre outros (Pinheiro et al., 2016).

Não obstante, para que todas essas possibilidades e demais procedimentos realizados nos repositórios educacionais sejam bem-sucedidos, faz-se necessário que estes possuam adequada usabilidade, a qual, de acordo com Nielsen (2012), é um atributo de qualidade que avalia como as interfaces são fáceis de serem utilizadas pelos usuários. Assim, a usabilidade colabora para o bom aproveitamento destes repositórios por instituições educacionais, professores e alunos. Nesse contexto, destaca-se o eduCAPES, um repositório educacional com OA abertos, instituído pelo Ministério da Educação do Governo Federal brasileiro em julho de 2016.

O presente estudo teve como foco analisar o eduCAPES, segundo alguns critérios de usabilidade, definidos a partir de uma análise documental, de maneira a oferecer informações referentes ao seu funcionamento, seus aspectos positivos e negativos, bem como verificar oportunidades de melhorias.

Esta pesquisa se deu a partir de uma atividade realizada em uma disciplina cursada no final de 2017, no Mestrado Profissional em Ensino e suas Tecnologias do IFFluminense, a qual se propunha a avaliar portais de objetos educacionais quanto sua facilidade de uso e dos seus OA. No desenvolvimento dessa pesquisa não foi encontrado nenhum trabalho científico, tanto na base de periódicos da CAPES como no Google Scholar, que realizasse um estudo específico sobre o eduCAPES e sua usabilidade, o que justifica, portanto, a relevância deste trabalho e sua contribuição para a comunidade acadêmica.

De modo a se alcançar o objetivo do trabalho, este artigo aborda, na próxima seção, a usabilidade em sistemas de informação. Na seção três, descreve-se o eduCAPES. A seção quatro traz a metodologia utilizada para o levantamento dos conceitos e critérios de usabilidade, conforme a literatura, enquanto na seção cinco são expostos os critérios de usabilidade definidos para a análise, bem como os resultados obtidos a partir desta. Por fim, na seção seis, são explanadas as considerações finais.

\section{Usabilidade em sistemas de informação}

A usabilidade se refere à adequação de um sistema às necessidades dos usuários, permitindo verificar o desempenho e a satisfação destes ao realizar determinadas tarefas (Bassani et al., 2010; Santos et al., 2014).

Para Nielsen (2012), a usabilidade é um atributo de qualidade que pode ser definida por cinco componentes: (i) capacidade de aprendizado: o sistema deve permitir que os usuários realizem facilmente tarefas básicas na primeira vez em que o utilizem; (ii) eficiência de uso: uma vez que os usuários tenham aprendido a utilizar o sistema, estes devem ser capazes de executar as tarefas com rapidez e produtividade; (iii) facilidade de memorização: o sistema deve possuir recursos que o tornem fácil de ser memorizado para que, depois de algum tempo sem utilizá-lo, os usuários se recordem 
de como ele funciona; (iv) baixa taxa de erros: o sistema deve prever e evitar que os usuários cometam erros e, caso estes ocorram, deve permitir uma rápida recuperação por meio de instruções e soluções simples; e (v) satisfação: o sistema deve possuir design e recursos que sejam agradáveis aos usuários.

De acordo com Miletto e Bertagnolli (2014), quando a construção de um sistema interativo é orientada pela usabilidade, pode se tornar mais fácil, precisa e agradável a realização de atividades pelos usuários. Caso contrário, o usuário necessitará de mais esforço para realizar suas tarefas, o que pode levar à subutilização ou ao abandono do sistema (Miletto; Bertagnolli, 2014).

Nos estudos realizados por Ieiri e Braga (2015), pôde ser verificado que muitos repositórios educacionais foram desenvolvidos visando apenas ao funcionamento de seu sistema, tendo pouca ou nenhuma atenção em relação à sua usabilidade. Os autores destacam, também, a carência de estudos no Brasil que abordem a usabilidade destes repositórios, indicando que o tema deve ser mais explorado (Ieiri; Braga, 2015).

\section{O repositório educacional eduCAPES}

O repositório eduCAPES (Figura 1), é classificado como um repositório educacional digital, tendo sido criado para ser um repositório de OA abertos, que pode ser usado tanto por alunos, como por profissionais de educação básica, graduação e pósgraduação. Seu acervo pode conter recursos, tais como laboratórios remotos e virtuais, jogos educacionais, e-books, videoaulas, dissertações, e quaisquer outros materiais de pesquisa e ensino, desde que estes sejam licenciados de maneira aberta ou sob domínio público e sejam provenientes do Sistema Universidade Aberta do Brasil (UAB) ou de parcerias com demais instituições que dispõem materiais educacionais (Brasil, 2016).

Figura 1 - Repositório educacional eduCAPES

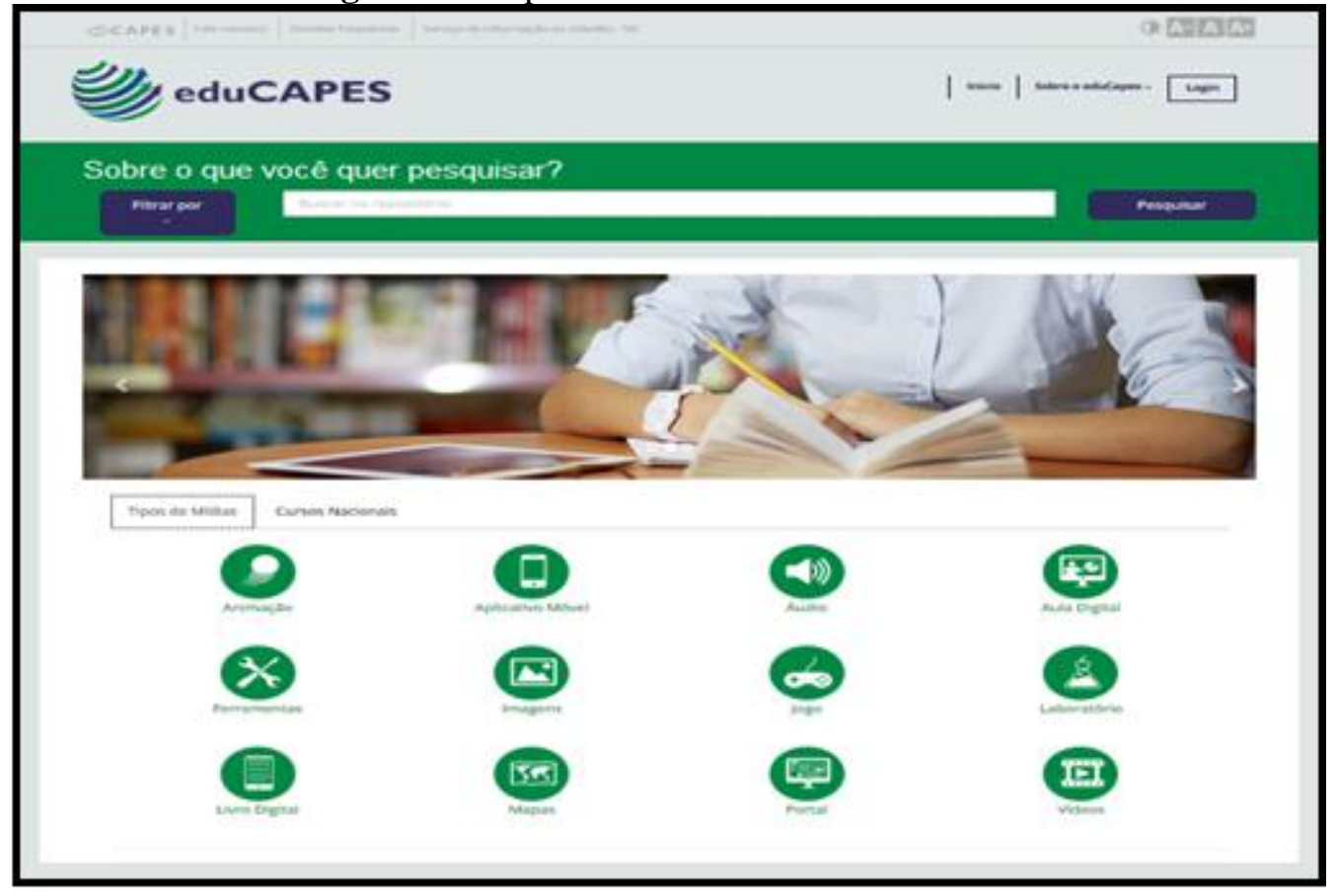

Fonte: https://educapes.capes.gov.br

Com relação à tecnologia utilizada, no site do eduCAPES, explica-se que este foi criado a partir do software livre DSpace, que busca prover acesso fácil e livre a vários tipos de materiais digitais. O DSpace foi desenvolvido com o objetivo de possibilitar a criação de repositórios digitais com funções de armazenamento, gerenciamento, 
preservação e visibilidade da produção intelectual, sendo facilmente adaptado às demandas das instituições (IBICT, 2018). Esta tecnologia também permite a integração com repositórios educacionais de outras instituições de ensino, desde que estas também utilizem o DSpace. Assim, o eduCAPES consente a inclusão de OA abertos que estejam mapeados em algum esquema de metadados compatível.

No eduCAPES, os OA são denominados materiais. Conforme pesquisa realizada no repositório em maio/2018, este contém mais de 107 mil OA publicados de maneira aberta, com autorização expressa do autor ou que estejam sob domínio público. Para pesquisar e fazer o download dos OA disponíveis não é necessário nenhum cadastro. Há, contudo, restrições para submissão do OA e a necessidade de cadastro no repositório. De acordo com o eduCAPES, somente participantes das universidades do programa UAB têm permissão para submeter OA. Atendendo a essa condição, além do cadastro, também é necessário possuir autoria ou licença de direitos autorais destes OA.

No próprio site do repositório há orientações sobre os tipos de licença Creative Commons sob a qual um OA pode ser licenciado e como proceder para licenciá-lo. A Creative Commons é uma organização sem fins lucrativos que utiliza instrumentos jurídicos gratuitos para permitir o compartilhamento e o uso da criatividade e do conhecimento (Creative Commons, 2018).

Já a responsabilidade dos OA submetidos é exclusiva do submissor e a análise do conteúdo destes é feita pelos próprios usuários do repositório, que podem avaliá-los e, em caso de irregularidade, denunciá-los. Também é possível encontrar no site um passo a passo com orientações sobre como realizar a submissão de OA e uma área de FAQ (Frequently Asked Questions - Perguntas Frequentes), que tem como propósito esclarecer as dúvidas mais frequentes em relação ao uso do eduCAPES.

No que diz respeito ao acesso aos OA existentes, este pode ser feito de forma híbrida: os OA podem estar hospedados no próprio eduCAPES ou em repositórios de instituições parceiras. Neste último caso, há um link na página de descrição do OA que direciona os usuários para os repositórios destas instituições. Para obter informações sobre os OA em repositórios de parceiros é feito um sincronismo de informações que permite a detecção automática de OA que foram adicionados ou removidos. Entre as instituições parceiras, estão o Banco Internacional de Objetos Educacionais (BIOE), o Khan Academy e o Instituto Nacional de Pesquisas Espaciais (INPE).

\section{Metodologia da pesquisa}

A presente pesquisa foi efetivada no âmbito do Mestrado Profissional em Ensino e suas Tecnologias, por quatro alunas, sendo três docentes e uma profissional da área de Tecnologia da Informação, sob a orientação da professora da disciplina de Ambientes Virtuais de Aprendizagem, que é especialista nesta área.

A escolha da análise do repositório educacional eduCAPES, pelo aspecto da usabilidade da interface, ocorreu devido a esta influenciar diretamente na sua utilização para a busca e seleção dos OA. A metodologia utilizada nesta pesquisa foi baseada na análise documental, tendo como objetivo identificar trabalhos que pudessem apresentar informações sobre os conceitos e critérios de análise da usabilidade em repositórios educacionais, para uma posterior análise utilizando estes critérios no eduCAPES. Para Gil (2008), a pesquisa documental é desenvolvida a partir de materiais já existentes, constituídos, sobretudo, por livros e artigos científicos. O autor expõe que essa pesquisa envolve materiais que não receberam tratamento analítico e que podem ser aperfeiçoados de acordo com os objetivos da pesquisa (Gil, 2008). Como bases de dados, utilizaram-se o Portal de Periódicos da CAPES e Google Scholar. 
No Portal de Periódicos da Capes, a busca foi realizada em dezembro de 2017, a partir dos descritores "usabilidade" e "repositório", utilizando-se como filtros, a data de publicação nos últimos cinco anos e o idioma dos artigos em português, obtendo-se, como retorno, um total de 26 artigos. Como critério, realizou-se a leitura dos títulos e resumos, sendo excluídos os artigos que não apresentavam clareza e foco nas características de análise da usabilidade de repositórios e os que não continham textos completos. A partir destes critérios, foi selecionado apenas um artigo: Lameira (2016).

Já no Google Scholar, com a finalidade de uma ampliação da temática, no mesmo período, foram utilizados como descritores: avaliação, repositórios, objetos de aprendizagem, repositórios educacionais, usabilidade e eduCAPES. Como critérios de seleção, também foram definidos artigos no idioma português e publicações feitas há até cinco anos. Assim, entre os textos, foram selecionados, como base teórica, os artigos de Ieiri e Braga (2015) e Pinheiro et al. (2016). Cabe ressaltar que, nas duas bases de dados, pesquisadas não foram encontrados artigos que apresentassem um estudo, especificamente, sobre o eduCAPES.

Após este levantamento bibliográfico, passou-se à eleição dos critérios de usabilidade para a análise do eduCAPES. A pesquisa realizada por Lameira (2016) mostrou a ausência de métodos padronizados para análise e avaliação de repositórios que pudessem consolidar dados para estudos comparativos. Assim, como há um contingente pequeno de estudos referentes às avaliações de repositórios educacionais, foram utilizadas, como base para a escolha dos critérios de análise, as metodologias de avaliação de repositórios propostas por Lameira (2016) e Pinheiro et al. (2016).

A metodologia de avaliação proposta por Lameira (2016) foi baseada na revisão da literatura sobre os temas "avaliação de repositórios" e "avaliação de sistemas de informação", com o propósito de se identificarem os critérios que aparecem com maior frequência na literatura para avaliar repositórios e aplicar esta avaliação a sete repositórios institucionais, buscando-se destacar seus principais aspectos positivos e negativos. Foram estabelecidas cinco categorias para os critérios de avaliação: metadados, visibilidade, políticas, usuários e institucionalização (Lameira, 2016).

Já a pesquisa realizada por Pinheiro et al. (2016) teve como objetivo analisar tanto os repositórios educacionais como os objetos de aprendizagem neles contidos. Para esta análise foram definidas as seguintes dimensões: propósito do repositório; temas/disciplinas, âmbito (departamental, institucional, regional, nacional ou internacional) e o setor/público de uso prioritário (Pinheiro et al., 2016).

Dessa forma, a presente pesquisa elencou 25 critérios para análise da usabilidade do repositório educacional eduCAPES, 14 deles adaptados a partir do trabalho de Lameira (2016) e 11 do trabalho de Pinheiro et al. (2016). A estes critérios, foram adicionados três outros, julgados relevantes pelas pesquisadoras, a partir da utilização do eduCAPES, perfazendo um total de 28 critérios de análise da usabilidade, que foram classificados de acordo com os cinco componentes de usabilidade propostos por Nielsen (2012), tratados na seção 2. Estes critérios, assim como sua análise, são apresentados no Quadro 1 da seção seguinte.

\section{Critérios de análise da usabilidade e resultados obtidos}

Os 28 critérios escolhidos para a análise da usabilidade do eduCAPES foram classificados e reorganizados de acordo com os cinco componentes passíveis de mensuração relativos à usabilidade definidos por Nielsen (2012), que são: capacidade de aprendizado, eficiência de uso, facilidade de memorização, baixa taxa de erros e satisfação. Estes critérios foram analisados pelas próprias pesquisadoras a partir da navegação no site do eduCAPES (Quadro 1). 
Quadro 1 - Critérios e resultados da análise da usabilidade do eduCAPES

\section{Critérios}

Análise

\section{Capacidade de aprendizado}

1. Há acessibilidade para usuários com deficiência? (Lameira, 2016).

Há funcionalidades que permitem alterar o contraste das cores e aumentar ou diminuir a fonte do texto.

2. As páginas do repositório estão padronizadas? (Lameira, 2016).

$\mathrm{O}$ layout de todas as páginas possui as mesmas cores, fontes e o logotipo padrão do site.

3. Há uma seção na página do repositório contendo informações sobre o mesmo? (Lameira, 2016).

O repositório oferece informações gerais do repositório na página "Sobre o eduCAPES".

4. São disponibilizados textos de ajuda para utilização do repositório? (Lameira, 2016).

No menu "Sobre o eduCAPES" há links para páginas com orientações de uso e o menu "Dúvidas frequentes" apresenta uma página de FAQ. Contudo, não há nenhuma informação sobre os "Cursos Nacionais", que é um recurso incluído recentemente.

5. É disponibilizado o mapa do site para Não. navegação? (Lameira, 2016).

6. Há orientações sobre como o usuário deve proceder para submeter e efetuar download de OA? (Lameira, 2016).

Não há orientações sobre como efetuar o download dos OA, mas há orientações sobre como realizar buscas e como submeter os OA no menu "Sobre o eduCAPES", nos links "Como faço minha busca?" e "Como submeto meu material?".

7. O repositório permite interfaces em outros Não. idiomas? (Lameira, 2016).

\section{Eficiência de uso}

8. Há uma política de indexação de Sim. Baseada no padrão Dublin Core. conteúdos? (Lameira, 2016).

9. Há controle de vocabulários para Não. Na submissão do OA o campo de publicação e categorização dos OA? "Tipo" não é obrigatório, assim como o de (Lameira, 2016). palavras-chaves. O próprio submissor é responsável pelo título e resumo do OA.

10. Há flexibilidade em relação a atalhos? (Lameira, 2016).

Sim. O menu superior está disponível em todas as páginas e, na maioria das páginas, é exibida a barra de busca.

11. Há algum texto orientando os usuários em como citar os OA? (Lameira, 2016).

Não.

12. É possível utilizar filtros variados para busca de OA? (Pinheiro et al., 2016).

Sim. Na barra busca principal é possível utilizar como filtros: assunto, autores, data do documento, título e material UAB. Após a busca inicial é possível utilizar outros filtros como: o repositório em que essa busca deve ser realizada, instituição, curso, tipo de 


\begin{tabular}{|l|l|}
\hline & arquivo, idioma, entre outros. \\
\hline $\begin{array}{l}\text { 13. Há indicação do público alvo destinado } \\
\text { para cada OA? (Pinheiro et al., 2016). }\end{array}$ & $\begin{array}{l}\text { Não. Os OA são destinados ao uso } \\
\text { educacional de forma geral. }\end{array}$ \\
\hline $\begin{array}{l}\text { 14. Há espaço para avaliação (comentários, } \\
\text { sugestões) dos OA? (Pinheiro et al., } \\
\text { 2016). }\end{array}$ & $\begin{array}{l}\text { Há como avaliar cada OA utilizando critérios } \\
\text { de a estrelas e realizar denúncias, de } \\
\text { forma sigilosa, em caso de irregularidades. } \\
\text { Contudo, não há como inserir comentários. }\end{array}$ \\
\hline $\begin{array}{l}\text { 15. Há indicação dos OA mais utilizados? } \\
\text { (Pinheiro et al., 2016). }\end{array}$ & Não. \\
\hline $\begin{array}{l}\text { 16. É fácil buscar os OA e acessá-los? } \\
\text { (Pinheiro et al., 2016). }\end{array}$ & $\begin{array}{l}\text { É fácil utilizar a opção de busca, que está } \\
\text { disponível em quase todas as páginas do } \\
\text { repositório e é possível utilizar vários filtros } \\
\text { de forma intuitiva. Já o download dos OA } \\
\text { apresenta maior complexidade, pois pode } \\
\text { direcionar a outros sites. }\end{array}$ \\
\hline $\begin{array}{l}\text { 17. Há informações pertinentes sobre os } \\
\text { metadados de cada OA? (Pinheiro et al., } \\
\text { 2016). }\end{array}$ & $\begin{array}{l}\text { Sim. Todos os OA possuem informações } \\
\text { básicas e ao clicar em "Mostrar registro } \\
\text { completo do item" é possível ver as } \\
\text { informações dos seus metadados. }\end{array}$ \\
\hline $\begin{array}{l}\text { 18. Há mecanismos para atrair contribuidores } \\
\text { e estimular a participação na manutenção } \\
\text { do repositório? (Pinheiro et al., 2016). }\end{array}$ & $\begin{array}{l}\text { Na página de "Dúvidas frequentes" há } \\
\text { instruções sobre como se tornar um parceiro } \\
\text { e como as instituições que desejam ser } \\
\text { parceiras podem disponibilizar seus OA. } \\
\text { Contudo, os contribuidores devem pertencer } \\
\text { à universidades do programa UAB. }\end{array}$ \\
\hline
\end{tabular}

Facilidade de memorização

19. O nome repositório aparece na URL? (Lameira, 2016).

Sim, facilitando a identificação da página e a memorização do endereço web.

20. O nome ou logotipo do repositório está sempre visível? (Lameira, 2016).

Sim. O nome e o logotipo constam no cabeçalho e no rodapé de todas as páginas.

21. O endereço web (URL) do repositório é de fácil digitação e/ou memorização? (Pinheiro et al., 2016).

Sim. Contém o nome do próprio repositório no endereço e a nomenclatura (domínio) utilizada para sites da CAPES (capes.gov.br).

\section{Baixa Taxa de Erros}

22. São disponibilizados meios de contato com os administradores do repositório (email, telefone, etc.)? (Lameira, 2016).

Sim. No menu "Sobre o eduCAPES" há a opção "Contato" que disponibiliza e-mail e telefone gratuito para contato, além de um link para a página "Fale Conosco" do MEC. Contudo, na barra superior há a opção "Fale conosco" do próprio repositório que requer login no sistema.

23. Na submissão dos OA há marcações sobre campos do formulário que são de preenchimento obrigatório? (Elaboração própria).

24. Na submissão dos OA há instruções sobre como preencher os campos do formulário?

Nem todos os campos de preenchimento obrigatório são destacados (com um asterisco).

Há diversas informações sobre como preencher o formulário de submissão de OA 


\begin{tabular}{|l|l|}
\hline (Elaboração própria). & $\begin{array}{l}\text { e há um link para uma página com } \\
\text { orientações mais detalhadas sobre como } \\
\text { submeter um OA. }\end{array}$ \\
\hline $\begin{array}{l}\text { 25. Em caso de preenchimento incorreto de } \\
\text { um campo do formulário, são } \\
\text { apresentadas mensagens de erro simples e } \\
\text { claras? (Elaboração própria). }\end{array}$ & $\begin{array}{l}\text { Sim. As mensagens de erro ou com } \\
\text { instruções de preenchimento aparecem } \\
\text { destacadas e são de fácil entendimento. }\end{array}$ \\
\hline \multicolumn{3}{|c|}{ Satisfação } \\
\hline $\begin{array}{l}\text { 26. As cores utilizadas são atraentes? } \\
\text { (Pinheiro et al., 2016). }\end{array}$ & $\begin{array}{l}\text { Não. O site utiliza, basicamente, as cores } \\
\text { verde e azul, em tonalidade forte, com o } \\
\text { fundo cinza. }\end{array}$ \\
\hline $\begin{array}{l}\text { 27. As imagens são nítidas? (Pinheiro et al., } \\
\text { 2016). }\end{array}$ & Sim. \\
\hline $\begin{array}{l}\text { 28. Há propagandas na página do repositório? } \\
\text { (Pinheiro et al., 2016). }\end{array}$ & $\begin{array}{l}\text { Não. São apresentadas apenas as instituições } \\
\text { parceiras. }\end{array}$ \\
\hline
\end{tabular}

Fonte: Elaboração própria

No componente "Capacidade de aprendizado", destacaram-se, como aspectos positivos, a padronização das páginas (item 2) e a disponibilização de diversas páginas com informações sobre como utilizar o repositório em questão (itens 3 e 4). Estes aspectos contribuem para que o usuário assimile rapidamente o funcionamento do sistema e consiga realizar tarefas básicas já na primeira vez em que for utilizá-lo. Como aspecto negativo, ressalta-se a inexistência de orientações sobre os procedimentos que devem ser realizados para efetuar o download dos OA (item 6), que, em alguns casos, direcionam o usuário para sites externos, tornando o acesso a estes mais complexo.

No componente "Eficiência de uso" verificou-se como principais aspectos positivos a política de indexação de conteúdos (item 8), a flexibilidade em relação aos atalhos (item 10) e a existência de filtros variados para a busca de OA (item 12). Tais aspectos facilitam a busca e a localização dos OA, além de otimizar o tempo gasto pelo usuário no repositório. Como aspectos negativos, destacaram-se a falta de controle de vocabulário para a publicação e categorização dos OA (item 9), a indicação do públicoalvo a que se destina cada OA (item 13) e a limitação na avaliação de cada OA (item 14). A ausência destas informações torna o processo de busca e seleção dos OA mais difícil e demorado, mesmo ambientando-se ao site, diminuindo sua produtividade.

Quanto ao componente "Facilidade de memorização", todos os itens foram considerados positivos. O fato de o nome e o logotipo do repositório estarem visíveis em todas as suas páginas (item 20) e o seu endereço web (URL) ser de fácil memorização (item 21) permite que o usuário reconheça e se recorde facilmente do site do repositório, mesmo depois de um determinado tempo sem utilizá-lo.

Em relação ao componente "Baixa Taxa de Erros", foram identificados, como aspectos positivos, a disponibilização de instruções sobre preenchimento no formulário para submissão de OA (item 24), como também as mensagens de erro serem claras e simples (item 25). Estes aspectos contribuem para que os usuários cometam poucos erros durante a utilização do sistema e, caso estes ocorram, permitem uma fácil correção. Um aspecto negativo a ser ressaltado é que nem todos os campos de preenchimento obrigatório são destacados (item 23), proporcionando uma maior probabilidade de erro ao preencher o formulário.

Por fim, quanto ao componente "Satisfação", destacou-se, como aspecto positivo, a ausência de propagandas (item 28), o que evita a poluição visual e contribui 
para uma utilização agradável do repositório. Como aspecto negativo nessa categoria estão as cores utilizadas (item 26), pois o site utiliza somente as cores verde e azul, em tonalidade forte, em um fundo cinza, tornando-o, visualmente, pouco atraente.

De acordo com os cinco componentes de usabilidade descritos por Nielsen (2012), o eduCAPES apenas não atendeu adequadamente o componente "Eficiência de uso", apesar de existir a necessidade de melhoria de alguns aspectos relativos aos demais componentes. Assim, apesar de a maioria dos critérios apresentarem aspectos positivos, constatou-se que mudanças podem ser implementadas, tendo em vista o desenvolvimento e aperfeiçoamento do repositório eduCAPES.

Como melhorias, sugere-se que haja um maior incentivo à utilização dos $\mathrm{OA}, \mathrm{o}$ que seria possível por meio da disponibilização de instruções sobre como efetuar o download e de informações mais detalhadas sobre os OA, tais como o seu objetivo e o seu público-alvo. Há também necessidade de aprimorar a forma de avaliação dos OA a partir de critérios como: adequação do conteúdo à proposta do $\mathrm{OA}$, adequação à linguagem utilizada ao o público-alvo, facilidade de utilização, além da possibilidade de inserir comentários.

Durante a utilização do repositório, verificaram-se diferenças, nos nomes dos campos dos formulários e dos menus, nas páginas atuais do repositório em relação às informações contidas nas páginas com instruções de uso. Logo, sugere-se uma revisão das páginas de instruções para sua adequação à configuração atual do repositório.

Outro aspecto a ser tratado é a implementação de algum mecanismo para controle do vocabulário ao se submeter um OA, como, por exemplo, a moderação do conteúdo por uma equipe do próprio repositório ou por meio de recursos tecnológicos, uma vez que as informações fornecidas na submissão impactam diretamente nos resultados das buscas pelos OA. O formulário de submissão de OA também deve ser revisto para a correta marcação dos campos que são obrigatórios.

Conforme já mencionado no aporte teórico, os estudos sobre repositórios educacionais visam muito mais a analisar as funcionalidades e não os requisitos de usabilidade, existindo uma carência de pesquisas nessa área. Assim, a partir dos resultados obtidos por meio da análise dos 28 critérios definidos, buscou-se mapear os aspectos positivos e negativos, bem como oportunidades de melhorias relativos à usabilidade do eduCAPES.

Desta forma, espera-se, com a análise efetuada, contribuir com a inclusão das melhorias propostas, potencializando a satisfação e à percepção da eficiência em relação ao uso do repositório educacional eduCAPES pela comunidade acadêmica.

\section{Considerações finais}

Com esse estudo, ficou evidente que o eduCAPES apresenta diversos aspectos vantajosos e recursos úteis para a área da educação, os quais contribuem para a pesquisa e o aprimoramento do conhecimento de educadores e alunos que buscam OA de acesso público, em vários formatos e para diversos níveis de ensino. No entanto, o repositório ainda necessita de alguns ajustes para ser utilizado de maneira mais eficiente, satisfatória e por um público maior. Acredita-se que a análise realizada pode contribuir para que o repositório seja aperfeiçoado a partir dos apontamentos e sugestões feitas.

Para trabalhos futuros, sugere-se o aprimoramento da análise da usabilidade do eduCAPES, levando-se em consideração uma maior amostra de usuários finais, a fim de contribuir para que os responsáveis por esse repositório educacional conheçam as necessidades de seus usuários e, a partir dessas informações, possam promover as melhorias necessárias nos serviços oferecidos. 


\section{Referências bibliográficas}

ALMEIDA, M. G.; FREITAS, M. C. D. F. Desafios permanentes: projeto político pedagógico, gestão escolar, métricas no contexto das TICs. Rio de Janeiro: Brasport, 2015.

BASSANI, P. B. S.; BEHAR, P. A; HEIDRICH, R. O.; BITTENCOURT, A.; ORTIZ, E. Usabilidade e acessibilidade no desenvolvimento de interfaces para ambientes de educação à distância. RENOTE - Novas Tecnologias na Educação, v.8, n.1, p.1-10, 2010.

BONOTTO, A. K.; BISOGNIN, E. Contribuições de um Objeto de Aprendizagem e dos Registros de Representações Semióticas no Estudo da Função Exponencial. RENOTE Novas Tecnologias na Educação, v.13, n.2, p.1-11, 2015.

BRASIL. Portaria n ${ }^{0}$ 106, de 14 de julho de 2016. Diário Oficial, Brasília, DF, 15 jul. 2016. Seção 1, p. 14. Disponível em:

<www.capes.gov.br/images/stories/download/legislacao/portaria-n-106-14-julho2016.pdf>. Acesso em: $10 \mathrm{dez} .2017$.

CREATIVE COMMONS. Sobre. Disponível em: 〈br.creativecommons.org/sobre〉. Acesso em: 30 abr. 2018.

EDUCAPES. FAQ - Perguntas Frequentes. Disponível em: <educapes.capes.gov.br/redirect?action=faq>. Acesso em: 30 abr. 2018.

GIL, A. C. Métodos e técnicas de pesquisa social. São Paulo: Atlas, 2008.

IBICT - Instituto Brasileiro de Informação em Ciência e Tecnologia. Sistema para Construção de Repositórios Institucionais Digitais (DSpace). Disponível em: $<$ www.ibict.br/pesquisa-desenvolvimento-tecnologico-e-inovacao/Sistema-paraConstrucao-de-Repositorios-Institucionais-Digitais>. Acesso em: 30 abr. 2018.

IEIRI, A. Y.; BRAGA, J. C. Problemas de Usabilidade em Repositórios de Objetos de Aprendizagem. In: SIMPÓSIO BRASILEIRO DE INFORMÁTICA NA EDUCAÇÃO, 26. 2015, Maceió. Anais. Maceió: CBIE, 2015, p.732-741.

LAMEIRA, A. K. A. Avaliação de repositórios institucionais brasileiros: Uma proposta de método de avaliação. Cadernos BAD, n.2, p.153-167, 2016.

MILETTO, E. M.; BERTAGNOLLI, S. de C. Desenvolvimento de Software II:

Introdução ao Desenvolvimento Web com HTML, CSS, Java Script e PHP. Porto Alegre: Bookman, 2014.

NIELSEN, J. Usability 101: Introduction to Usability. Fremont: Nielsen Norman Group, 2012. Disponível em: <www.nngroup.com/articles/usability-101-introduction-to-usability>. Acesso em: 30 dez. 2017.

PINHEIRO, A. C.; RUMENOS, N. N.; TEZANI, T. C. R. Repositórios de objetos de aprendizagem no ensino de ciências e matemática: uma breve análise. Infor, Inov. Form., Rev. NEaD-Unesp, São Paulo, v.2, n.1, p.266-288, 2016.

SABBATINI, M. Reflexões críticas sobre o conceito de objeto de aprendizagem aplicado ao ensino de ciências e matemática. EM TEIA - Revista de Educação Matemática e Tecnológica Iberoamericana. v.3, n.3, p.1-36, 2012.

SANTOS, D.B.; MOURA, A. M. M.; PAVÃO, C. M. G. Proposta metodológica para avaliação de usabilidade de interfaces de repositórios. Cadernos BAD, n.2, p.215-218, 2014. 